\title{
ANN Model of RF MEMS Lateral SPDT Switches for Millimeter Wave Applications
}

\author{
S.Suganthi ${ }^{* 1}$, K.Murugesan ${ }^{* * 2}$ and S.Raghavan ${ }^{* * * 3}$ \\ 1. Research Scholar, 2. Vice Principal 3. Professor, \\ Department of Electronics and Communication Engineering, \\ 1. Anna University, Coimbatore, 2. Sree Sastha Institute of Engineering and Technology, Chennai \\ 3.National Institute of Technology, Tiruchirappalli -620015, \\ Tamilnadu, INDIA. \\ Email: sugi19770@yahoo.com
}

\begin{abstract}
This paper presents Artificial Neural Network (ANN) implementation for the Radio Frequency (RF) and Mechanical modeling of lateral RF Micro Electro Mechanical System (MEMS) series micro machined Single pole double through (SPDT) switch. We propose an efficient approach based on ANN for analyzing the losses in $\mathrm{ON}$ and OFF state of lateral RF MEMS series switch by calculating the S-parameters. The double beam structure has been analyzed in terms of its return, isolation and insertion losses with the variation of its passive circuit component values. The effect of design parameters has been analyzed and the lateral switch was realized with low insertion loss, high return and isolation losses. ANN model were trained with five different training algorithms namely LevenbergMarquart (LM), Bayesian Regularization (BR), Quasi Newton (QN), Scaled Conjugate Gradient (SCG) and Conjugate Gradient of Fletcher - Powell (CGF) to obtain better performance and fast convergence. The results from the neural model trained by Levenberg-Marquardt back propagation algorithm are highly agreed with the theoretical results available in the literature. The neural networks shows the better results with the highest correlation coefficient which measures the strength and direction of linear relation between two variables (actual and predicted values) $(0.9998)$ along with lowest root mean square error (MSE) of (0.0039).
\end{abstract}

Index Terms - Artificial neural networks, Micro electro mechanical systems, Training algorithms, correlation coefficient.

\section{INTRODUCTION}

Micro Electro Mechanical System (MEMS) is the integration of mechanical elements, sensors, actuators and electronics on common silicon substrate by micromachining process [1]. The advancements in the field of designing sensors, micro machines and control elements have facilitated much attention in the rapid developments of radio frequency (RF) MEMS. The first MEMS switch was demonstrated in 1971 using electrostatic actuation used to switch low frequency electrical signals 
[2]. When compared to the performance of MEMS switches, traditional integration of PIN diode and GaAs FET in switching circuits are degraded because of high insertion and low isolation losses in high frequency $(\mathrm{GHz})[3]$ range. They are suffering from high power consumption and significant inter modulation product due to nonlinear characteristics [4].

On the other hand, the recently developed RF MEMS switches exhibit excellent switching characteristics over wideband from RF to mm-wave frequencies $(0.1$ to $100 \mathrm{GHz})$ with the following remarkable advantages: extremely low insertion loss $(0.1 \mathrm{~dB})$ and very high isolation up to $100 \mathrm{GHz}$, near zero power consumption (10-200nJ/switching cycles), simple biasing circuit, potential for low cost and no inter modulation product (30-50dB better than PIN or FET switches) [4,5].

These superior qualities of RF MEMS switch make them viable structure for various applications such as signal routing in transceivers applications [8], phase shifters in phase array antenna [3], impedance matching networks [6], wide band tuning networks, reconfigurable antennas, filters and related circuits [9]. MEMS concepts have been successfully applied in the development of RF switches to be used in the phase shifters which are having the benefits of low loss, low parasitic and high linearity [10].

Based on direction of motion, MEMS switches can be classified into two main categories: vertical and lateral switches. Most of the reported works are based on vertical switch which performs in wafer plane displacement and surface contact [6]. But the lateral switch performs in wafer plane displacement and side wall contact. The vertical switches have the drawbacks of stiction problem during the moveable structure release. But in the case of lateral switches, the actuators, contacts, support structures and conducting paths can be fabricated in a single step and hence have the benefit of co-fabrication. Besides, it is easy to get mechanical force in opposing direction even when electrostatic designs are used $[6,7]$.

In microwave and millimeter wave communication systems, MEMS single pole double through (SPDT) switches are used as an alternative of GaAs MESFET and PIN diode integrated switching circuit [11] as they suffer from low isolation and high insertion loss. The MEMS SPDT switches are more suited for giga hertz $(\mathrm{GHz})$ range due to their low insertion, high isolation, negligible power consumption and good linearity.

Traditionally in design process, the objective of reducing the time and cost is relied on experiments. Since the usage of RF MEMS devices in modern IC (Integrated Circuits) and RF applications has been increased, an accurate and efficient Computer Aided Design (CAD) tools are required. The present full wave techniques for RF MEMS devices like FEM (Finite Element method) [12] and Finite Difference Time Domain (FDTD) method shows good accuracy with large memory requirements. The inability of these methods for RF MEMS modeling on system level limits their use for individual device level analysis. Though many approaches for circuit level simulation have been proposed, the design and optimization of electronics incorporating MEMS devices are still problematic. As an alternative for above mentioned limitations, ANN has been recognized as a viable 
modeling technique with massively parallel computation process. An accurate device characterization and efficient prediction of general input-output relationship can be achieved based on nonlinear mapping capabilities of neural network. Although extensive time and effort are required for preparing the dataset, once the network is trained, the proposed model accurately predicts the device responses for arbitrary inputs within the desired range.

In this work, a coplanar waveguide (CPW) lateral micro machined (SPDT) switching circuit which find application in high frequency transmitting and receiving signal routing is implemented using neural networks. Generation of training and testing datasets are realized from MATLAB simulation. Due to the optimization of generalized dimension of actuation part, the S-parameters of open and closed state of RF lateral switch and low actuation voltage are obtained. The resultant input and output relationship are mapped using the neural model. Based on valid range of input parameters, neural network are trained and tested. The neural models are trained with Levenberg-Marquart (LM), Bayesian Regularization (BR), Quasi - Newton (QN), Scaled Conjugate Gradient (SCG) and Conjugate Gradient of Fletcher - Powell (CGF) training algorithms.

\section{THEORY OF SINGLE AND DOUBLE BEAM LATERAL RF MEMS SWITCH}

In our previous work, the detailed loss analysis of single beam lateral (only one beam displaces) switch was discussed for ON and OFF states [13]. Additionally in this work, double beam (both beams are displaceable) RF lateral switch which provides a perfect RF symmetrical circuit has been analyzed. The double beam structure presents the advantages of lower insertion loss and high power handling and higher life time due to less residual stress. Since the spring constant for structures of single and double membranes are equal, the switching time for both structures will be the same. Actually the spring constant of the switch affects the threshold voltage. The spring constant of a membrane depends on the geometry, material, residual stress and degrees of its freedom. The only difference between the conventional single beam and double beam switch is an improvement on threshold voltage. This goal has been concentrated in this paper by controlling the original gap between the two electrodes and the width of beam and mass part of cantilever structure. The proposed double beam switch consisting of a Si-core finite ground coplanar waveguide (FGCPW) and an electrostatic actuator is shown in fig.1 (a) \& (b). The FGCPW is formed by thick single-crystal-silicon plate that has been coated with thin layer of aluminum (Al) to make the RF signal propagation not only along the metal on the top surface, but also on the sidewalls of the transmission line. In this switch, two cantilever beams are employed and can be used as signal lines together to propagate RF signal [14]. The fixed connections of the two cantilever beams are from the same port and the two contact tips are on the other port. At the free-end of the two cantilever beams, both ground lines extend towards the nearby cantilever beams to serve as their fixed electrodes respectively. 
When sufficient DC bias voltage is applied between the cantilever beam and the ground line, the cantilever beam is pulled toward the fixed electrode by electrostatic force until its free end hits the contact bump, resulting in the on-state of the switch. When DC bias voltage is removed, the mechanical stress of the beam overcomes the stiction forces and pulls the cantilever beam away, resulting in the off-state of the switch. Due to the asymmetrical layout of the two ports, the $S$ parameters obtained from the two ports are not reciprocal [3].

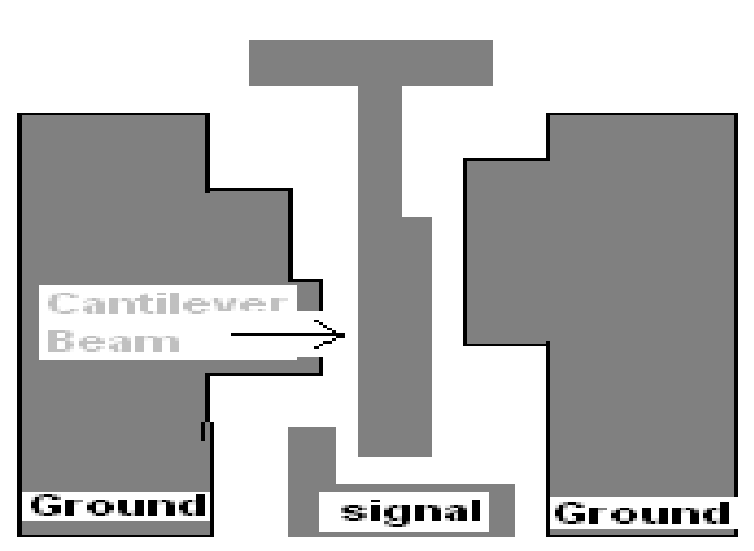

(a)

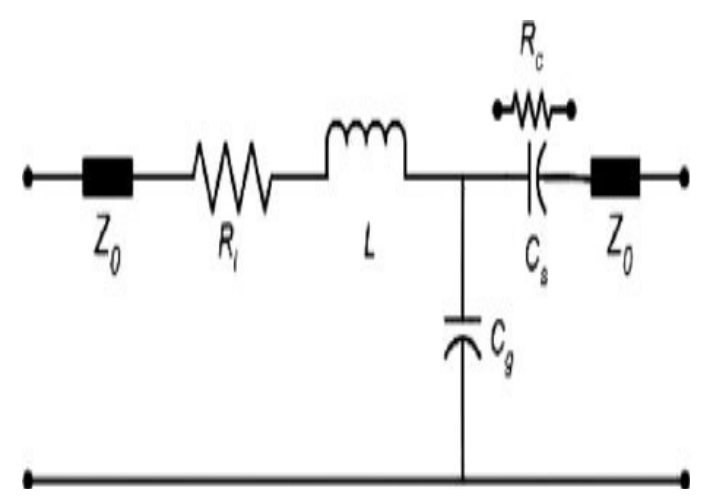

(c)

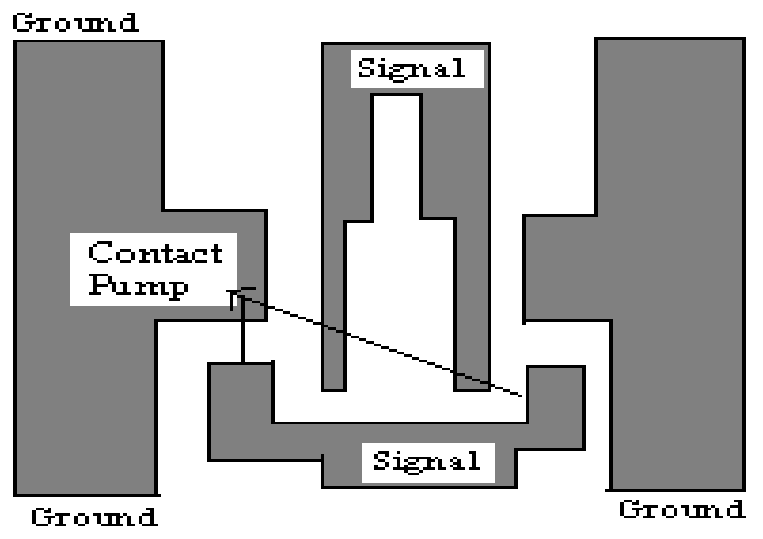

(b)

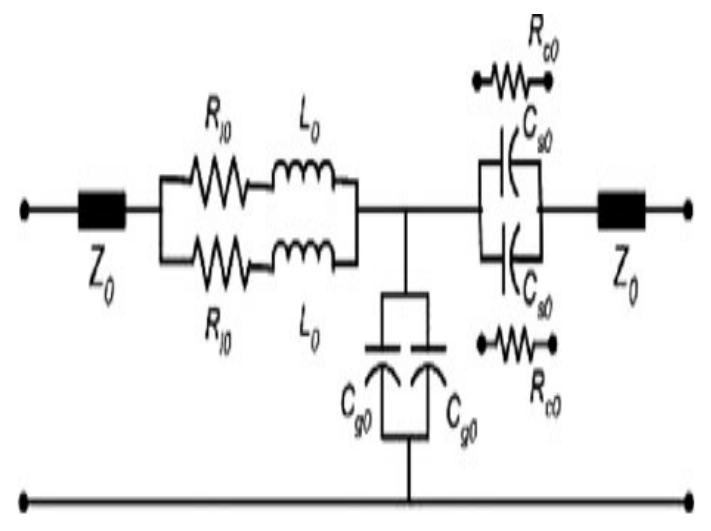

(d)

Fig.1. Schematics top view of RF lateral switches. (a) Single beam (b) Double beam. Equivalent electrical model circuits (c) Single beam (d) Double beam

\section{A. $R F$ modeling of the lateral SPDT switch}

An equivalent circuit model for the single beam RF MEMS switch is shown in fig 1(c). The model consists of characteristic impedance $Z_{0}$ of the input and output sections of the FGCPW transmission line, the resistor $R_{l}$ and the inductor $L$ of a cantilever beam, switch series capacitor $C_{s}$ (open-state), or contact resistor $R_{c}$ (closed state), and a shunt coupling capacitor $C_{g}$. In the single-beam switch, only one cantilever beam is used to serve as a movable electrode and signal line. In order to achieve low insertion loss and high power handling, double cantilever beams can be used as signal lines together to propagate RF signal. 
The equivalent circuit for the double-beam switch shown in fig.1(d) can be reduced to the from the equivalent circuit model of the single beam switch. Therefore, for the double beams switch, the circuit parameters are related with the electrical equivalent model of single beam switch as follows: the whole beam resistor and contact resistor of closed switch $\left(R_{1}=R_{10} / 2, R_{c}=R_{c 0} / 2\right)$, inductor $\left(L=L_{0} / 2\right)$, [3] series capacitor of open switch and shunt coupling capacitor $\left(C_{s}=2 C_{s 0}, C_{g}=2 C_{g 0}\right)$, with the assumption that the two cantilever beams are identical [14]. Hence, only the circuit model of the single beam switch is discussed in this paper.

In the open state, the switch performance is determined by the switch capacitance $C_{s}$. The shunt coupling $C_{g}$ between the cantilever beam and the fixed electrode can be estimated as

$$
C_{g}=\frac{\varepsilon_{0} l_{2} t}{g}+C_{f}
$$

Where $\varepsilon_{0}$ is the permittivity of the air $\left(8.854 \times 10^{-12} \mathrm{~F} / \mathrm{m}\right), l_{2}$ is the length of the electrode part of the cantilever beam, $t$ is the thickness of the beam, $g$ is the distance of the gap between two electrodes and $C_{f}$ is the fringing field capacitance. This coupling capacitance is fairly large and affects the loss mechanism in the closed state of the switch.

\section{B. Mechanical modeling of lateral switch}

When a micro machined circuit is designed, it is important to consider the switching voltage. The low actuation voltage can be achieved through the optimization of the geometrical dimensions of the actuation part. The top view of the electrostatic actuator used in the modeling is shown in fig.2. The actuator consists of four components: a suspended cantilever beam serving as a movable electrode, an anchor on the substrate to support the cantilever beam, a fixed electrode opposite to the cantilever beam and a contact bump.

The cantilever beam OC is a beam-mass structure. For the beam part OA, the width is $w_{1}$ and the length is $l_{1}$. For the mass part AC, the width is $w_{2}$ and the length is $\left(l_{2}+l_{3}\right)$ in which $l_{2}$ is the length of the electrode section $\mathrm{AB}$ and $l_{3}$ is the length of $\mathrm{BC}$. The mass width, $w_{2}$, is designed to be relatively wider than the beam width, $w_{1}$, so that low threshold voltage can be maintained and greater deformation of the electrode section may be avoided.

By assuming the electrode part of the cantilever beam subjected to a uniform load, the equivalent stiffness $\mathrm{k}$, of the cantilever beam [15] can be derived as the expression

$$
\begin{aligned}
k & =\frac{12 E_{1} E_{2} I_{1} I_{2}}{A E_{1} I_{1}+B E_{1} I_{2}} \\
A & =\left(\frac{3}{2} I_{2}^{3}+2 I_{2}^{2} I_{3}\right) \\
B & =\left(4 I_{1}^{3}+9 I_{2} I_{1}^{2}+6 I_{I} I_{2}^{2}+6 I_{1}^{2} I_{2}+6 I_{1} I_{2} I_{3}\right)
\end{aligned}
$$




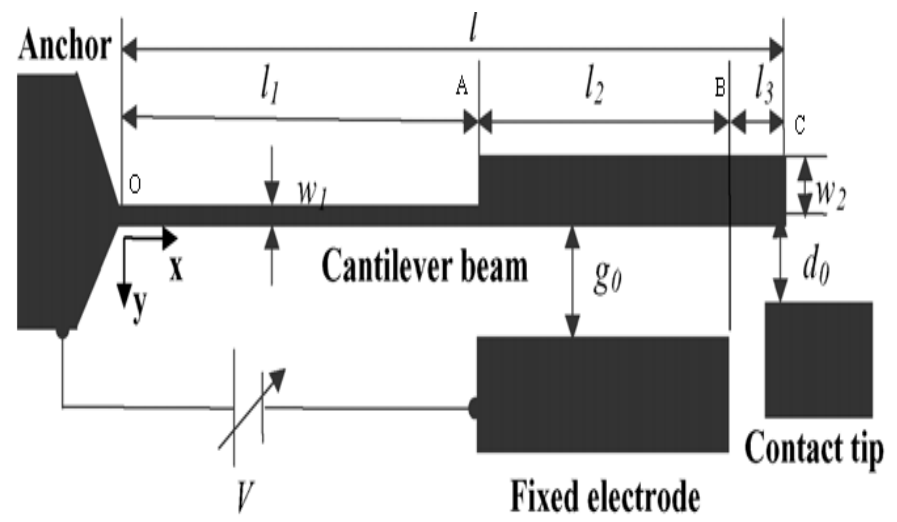

Fig.2. Top view of the electrostatic actuator

where $E_{1}$ and $E_{2}$ are the Young's moduli of the narrow part and wide part respectively. $I_{1}$ and $I_{2}$ are the moments of inertia of the cross sectional area of the narrow and wide part of the beam respectively. Before the deposition of the metal, the beam is merely made up of single-crystalsilicon. $E_{1}, E_{2}, I_{1}$ and $I_{2}$ is the Young's modules of the single crystal silicon and is given by $E_{1}=E_{2}=140 G P a$.

$$
\begin{aligned}
& I_{1}=\frac{1}{12} w_{1}^{3} t \\
& I_{2}=\frac{1}{12} w_{2}^{3} t
\end{aligned}
$$

After the deposition of $A l$ on the top surface of the beam, the beam is made of single crystal silicon partially covered with Al. Therefore $E_{1}, E_{2}, I_{1}$ and $I_{2}$ can be given by

$$
\begin{gathered}
E_{1}=\frac{E_{s i} w_{1}+2 E_{A l} w_{A l}}{w_{1}+2 w_{A l}} \\
I_{1}=\frac{1}{12}\left(w_{1}+2 w_{A l}\right)^{3} t \\
E_{2}=\frac{E_{s i} w_{1}+2 E_{A l} w_{A l}}{w_{2}+2 w_{A l}} \\
I_{2}=\frac{1}{12}\left(w_{2}+2 w_{A l}\right)^{3} t
\end{gathered}
$$

where $E_{A l}$ is the Young's modules of $\mathrm{Al}(70 \mathrm{GPa}), w_{A l}$ the thickness of $\mathrm{Al}$ deposited at sidewalls of the silicon beam.

For the design of the lateral switch in static behavior, low threshold voltage is always desired. The threshold voltage can be determined by above two forces. In this work, the threshold voltage is determined based on the cantilever beam structure by considering the original gap between two electrodes, the length ratio and the beam and mass width of the cantilever. 
Since the switching is carried out by an electrostatic force, one has to know the relation between the electrostatic force $F_{e}$ and the real-time distance $g$ of the gap between the ends of two electrodes. $F_{e}$ can be written as:

$$
F_{e}(g)=\frac{\varepsilon_{0} I_{2} t V^{2}}{2 g^{2}}
$$

where $V$ is the applied voltage. At equilibrium, the electrostatic force $F_{e}$ is equal to the restoring force $F_{r}$, which can be written as:

$$
F_{r}(g)=k\left(g_{0}-g\right)
$$

where $g_{0}$ is the initial gap between the two electrodes. The relation between $g$ and the applied voltage $V$ can be obtained by solving the following equation: $F_{r}(g)=k\left(g_{0}-g\right)$

\section{III.ARTIFICIAL NEURAL NETWORKS}

ANNs are biologically inspired computer programs to simulate the way in which the human brain process information. It is a very powerful approach for building complex and nonlinear relationship between a set of input and output data. The recent work by researchers demonstrated the ability of neural networks to learn and model a variety of microwave components, such as micro strip interconnects [16], packing and interconnects, spiral conductors and CPW components. Neural models can be much faster than original detailed EM/physics models, more accurate than polynomial and empirical models, allow more dimensions than table lookup models and are easier to develop when a new device / technology is introduced [17]. The cost for developing neural models is mainly depending on data collection and training.

The power of computation is determined from connections in a network. Each neuron has weighted inputs, simulation function, transfer function and output. The weighted sum of inputs constitutes the activation function of the neurons. The activation signal is passed through a transfer function which introduces non-linearity and produces the output. During training process, the inter-unit connections are optimized until the error in prediction is minimized. Once the network is trained, new unseen input information is entered into the network to calculate the test output. Many types of neural networks developed for various applications are available in the literature.

The class of neural network and its architecture for a particular model implementation depends on the nature of problem to be solved. The neural network architecture used in this paper is the MultiLayer Perceptron Neural Network (MLPNN). These networks having multilayer feed forward architecture composed of layers of computing nodes called neurons [16]. The MLPNN is one of the most extensively used ANN due to its well-known general approximation capabilities and limited complexity.

The MLPNN model used in this work consists of three layers: an input layer, an output layer and two intermediate or hidden layers. Each neuron in the input layer is acting only as a buffer for 
distributing the input signals $x_{i}$ to neurons in the hidden layer. Each neuron $j$ in the hidden layer sums up its input signals $x_{i}$ after weighting them with the strengths of the respective connections $w_{i j}$ from the input layer and compute its output $y_{j}$ as a function of the summation and

$$
Y_{j}=f\left(\sum W_{i j} x_{i}\right)
$$

where $f$ can be a simple threshold function, a sigmoidal [17] or hyperbolic tangent function [18]. The output of neurons in the last (output) layer is computed similarly.

Training a network consists of adjusting weights of the network using learning algorithms. During learning process, neural network adjusts the weights and thresholds so that the error between neural predicted output and sampled output is minimized. All learning algorithms used in this work are based on multilayer correction [19] learning algorithm called back propagation. The five different training algorithms we used in this work are described briefly as below.

\section{A. Levenberg - Marquardt (LM) algorithm}

This is a least -square estimation method based on the maximum neighborhood idea [20]. The LM method combines the best features of the Gauss-Newton technique and the steepest-descent method, but avoids many of their limitations. In particular, it generally does not suffer from the problem of slow convergence.

\section{B. Bayesian Regularization (BR) Algorithms}

This algorithm updates the weight and bias values according to their LM optimization and minimizes a linear combination of squared errors and weights, and then determines the correct combination so as to produce a well generalized network. This algorithm can train any network as long as its weight, inputs and transfer functions have derivative functions [21].

\section{Quasi-Newton (QN) Algorithm}

This is based on Newton's method but doesn't require calculation of second derivatives. An approximate Hessian matrix is updated. At each iteration of the algorithm, the update is computed as a function of the gradient. The line search function is used to locate the minimum [22]. The first search direction is the negative of the gradient of performance. In succeeding iterations the search direction is computed according to the gradient.

\section{Conjugate Gradient of Fletcher-Reeves (CGF)}

This method updates weights and bias values according to the conjugate gradient with FletcherReeves. Each variable is adjusted to minimize the performance along the search direction. The line search is used to locate the minimum point. Fletcher-Reeves version of conjugate gradient uses the norm square of previous gradient and the norm square of the current gradient to calculate the weights and biases [22]. 


\section{E. Scaled Conjugate Gradient (SCG) algorithm}

This algorithm can train any network as long as its weights, net inputs and transfer functions have derivative function. This algorithm is based on conjugate directions but does not perform line search at each iteration. This was design to avoid the time consuming line search.

\section{IV.PROPOSED ANN MODEL}

Two back propagation feed forward ANN architectures (ANN-RF for RF and ANN-MECH for mechanical modeling) are utilized in this work and are shown in fig 3(a) \& (b). All model parameters are allowed to vary and MATLAB simulation is used to generate the datasets for ANN models. The circuit parameters of RF model are selected as input and insertion and return loss are the outputs for ANN-RF while length ratio and initial gap distance are input and threshold voltage is output for ANN-MECH.

The selected ranges of input parameters are given as follows: $20 f F \leq C_{g} \leq 125 f F$, $10 P H \leq l \leq 300 P H, 1 \Omega \leq\left(R_{1}+R_{2}\right) \leq 10 \Omega, 1 G H z \leq f \leq 26 G H z$ and $w_{2}=5 \mu m, 1.8 \mu m \leq w_{1} \leq 5 \mu m, h=35 \mu m$, $0 \leq\left(l_{1}+l_{2}\right) \leq 1,4 \mu m \leq g_{0} \leq 8 \mu m \& w_{A l}=0.6 \mu m$ for ANN-RF and ANN-MECH respectively. The ratio of training to test data records employed is 70:30. This means that with 384 data records, there are 269 records for the training set and 115 records for the test set. The Levenberg-Marquardt algorithm uses input vectors and corresponding target vectors to train the neural networks. The number of hidden units directly affects the performance of the network. Therefore, many trails are conducted and the number of hidden nodes determined to provide the optimal result are 8 and 12 for first and second hidden layers of ANN-RF and ANN-MECH respectively.

Finally, the architecture of network is 5-8-8-2 for ANN-RF and 6-12-12-1 for ANN-MECH. The number of input nodes is $5 \& 6$ for ANN-RF and ANN-MECH respectively, representing the geometrical parameters of the switch that affect outputs. The number of the hidden nodes is set to 8 and 12 and the number of output nodes are $2 \& 1$ for ANN-RF and ANN-MECH respectively.

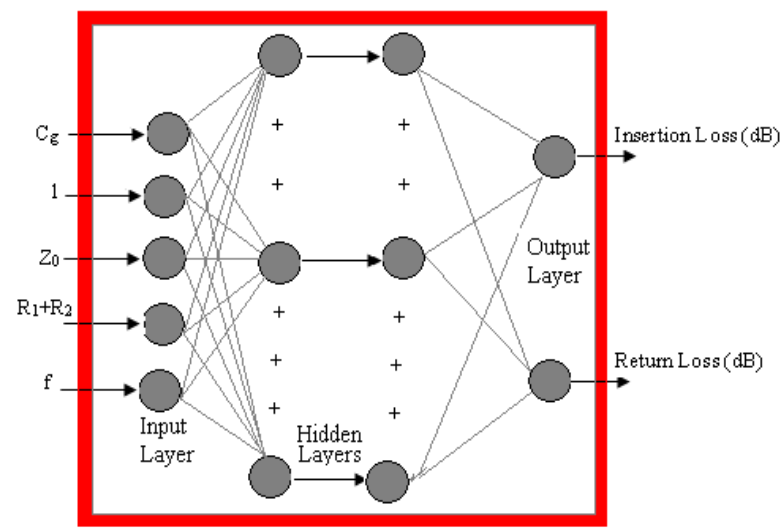

(a)

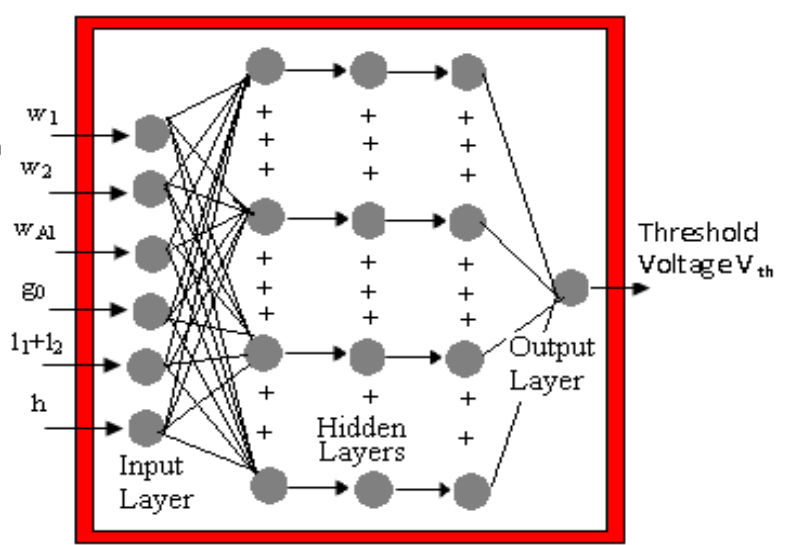

(b)

Fig.3. Feed forward ANN architecture with input-output parameters for (a) RF (b) Mechanical modeling 
In order to evaluate the performance of the ANN models, the mean square error (MSE) and the correlation coefficient $\left(R^{2}\right)$ as defined below are calculated in terms of the difference between the output of ANNs and training datasets.

$$
\begin{aligned}
& \text { MSE }=\frac{1}{N} \sum_{x=1}^{N}\left(\hat{x}_{i}-x_{i}\right)^{2} \\
& R^{2}=1-\frac{\sum_{i=1}^{N}\left(\hat{x}_{i}-x_{i}\right)^{2}}{\sum_{i=1}^{N}\left(x_{i}-\bar{x}_{i}\right)}
\end{aligned}
$$

where $\mathrm{N}$ is the total number of data sets, $\hat{x}_{i}$ is MATLAB dataset, $x_{i}$ is trained ANN output and $\bar{x}_{i}$ is mean of $x_{i}$.

\section{RESULTS AND DISCUSSION}

To obtain better performance, faster convergence and a simpler structure, the proposed ANN as shown in fig. 3 was trained with five different training algorithms. Figure.4(a) shows the RMS error comparison of five training algorithms for the calculation of S-parameters. From figure 4(b), it is clear that the best results were obtained from the models trained with LM algorithm.

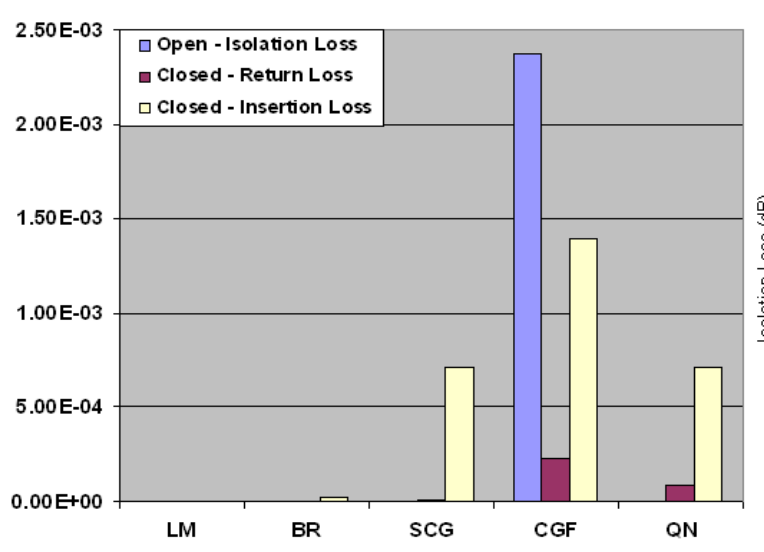

(a)

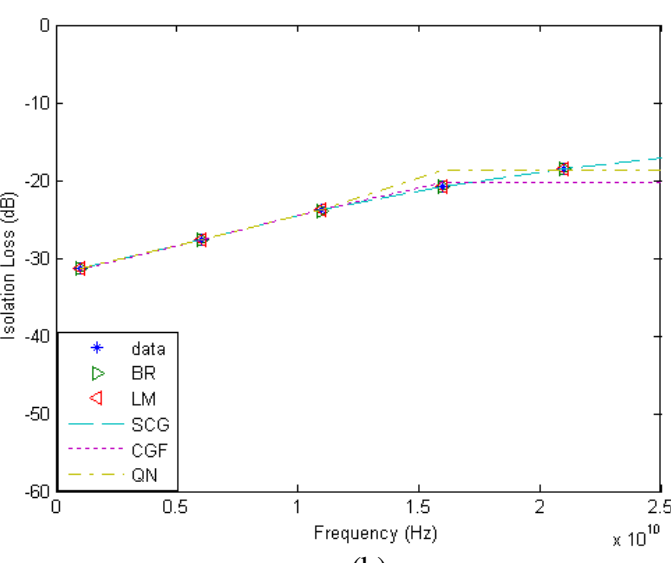

(b)

Fig.4. Comparison of training algorithms result (a) Bar chart of RMS error of training algorithms.(b)Isolation loss trained by different algorithms

To prove the efficiency and accuracy of the developed ANN models, the selected range of input values are used and the networks are validated. The comparison of predicted S-parameters from the networks and the simulated results are shown in fig 5(a) for open state, and (b) \& (c) for closed state respectively.

Fig 5 (a) shows that in the open state, the isolation of the switch increases with the decrease in $C_{s}$. Fig 5 (b) and (c) indicates the RF performances of the switch in terms of return and insertion loss of the closed state. It is clear that the performances are improved when $C_{g}$ increases from 10 to $60 \mathrm{fF}$. The performance of the closed switch begins to deteriorate when $C_{g}$ is increasing further to $125 \mathrm{fF}$. When $C_{g}$ is $60 \mathrm{fF}$, the insertion loss and the return loss of the switch get to their optimal value since a 
resonance occurs at the operating frequency range and the losses only depend on the total resistance of the circuit.

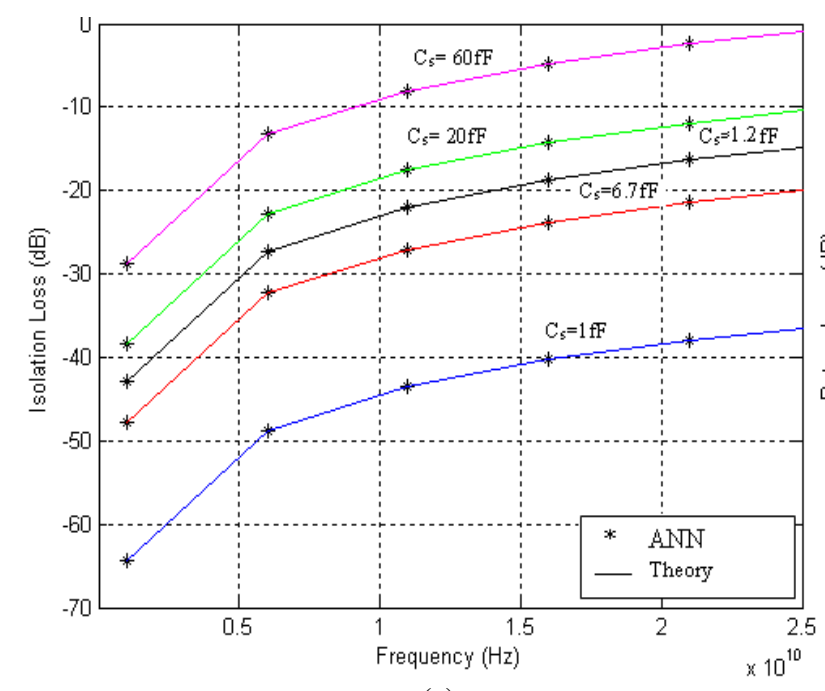

(a)

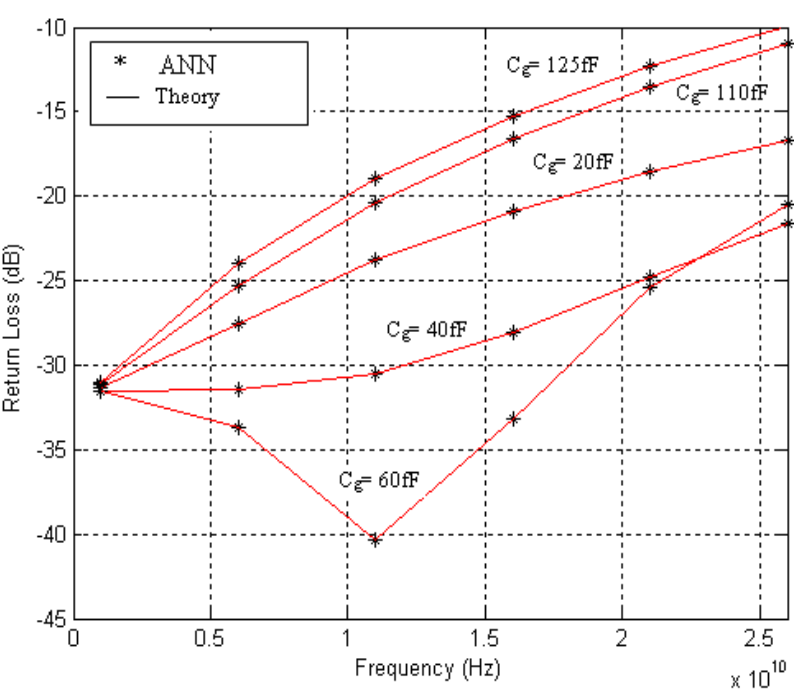

(b)

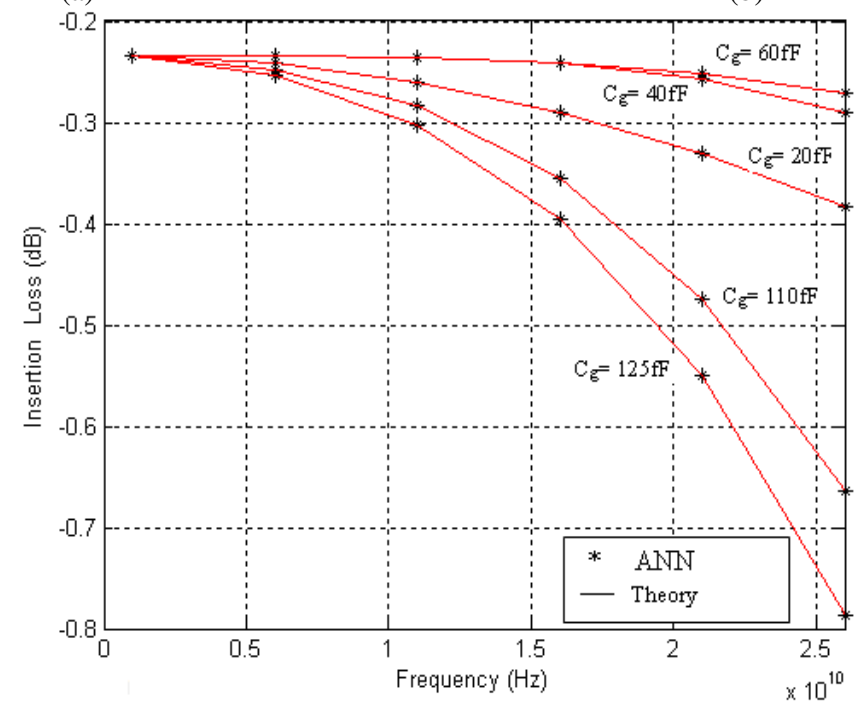

(c)

Fig.5.Comparision of ANN and simulation results of S-parameters for RF modeling (a) Open (b) Closed state return loss and (c) Insertion loss of lateral single beam switch with various capacitances $C_{g}$.

Figure 6(b) shows that that the threshold voltage increases as the initial distance between the two electrodes increases. It is seen that the threshold voltage is highly dependent on $w_{1}$, the width of the narrow part of the beam as compared to $w_{2}$, the width of wide part and $w_{A l}$, thickness of metal deposited.

When the value of $w_{1}$ is lesser than $w_{2}$, the effect of wide part is negligible in $V_{t h}$. For a given value of $w_{2}$, threshold voltage $V_{t h}$ is higher for the beam when $\mathrm{Al}$ is deposited compare to the case without $\mathrm{Al}$ deposition. Such a result is observed up to $w_{1}=3.8 \mu \mathrm{m}$. The threshold voltage $V_{\text {th }}$ is low in $\mathrm{Al}$ deposited beams above the width of $3.8 \mu \mathrm{m}$. This is due to the fact that RF signal propagation occurs not only along the metal on the top surface, but also on the sidewalls of the transmission line. 


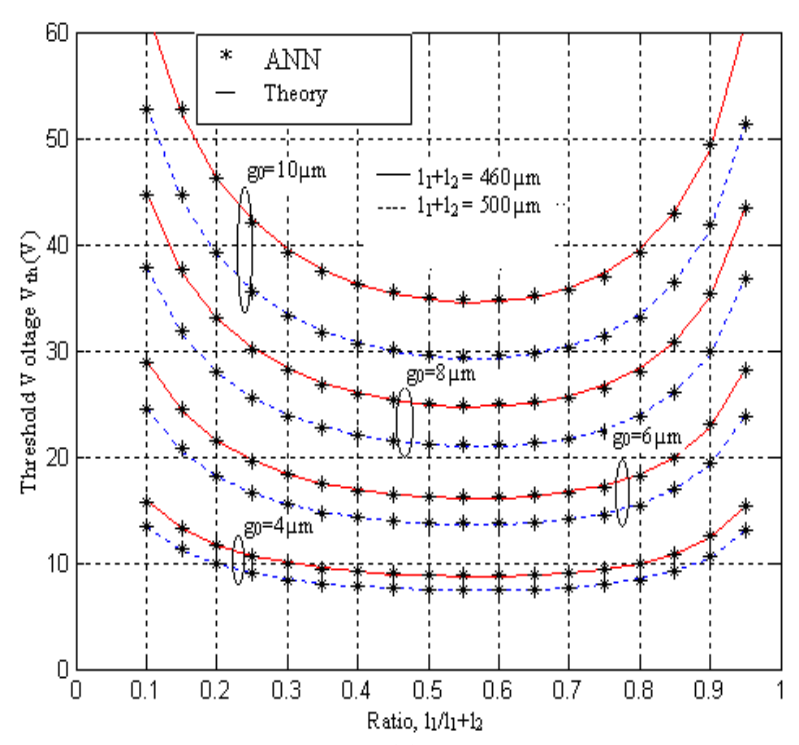

(a)

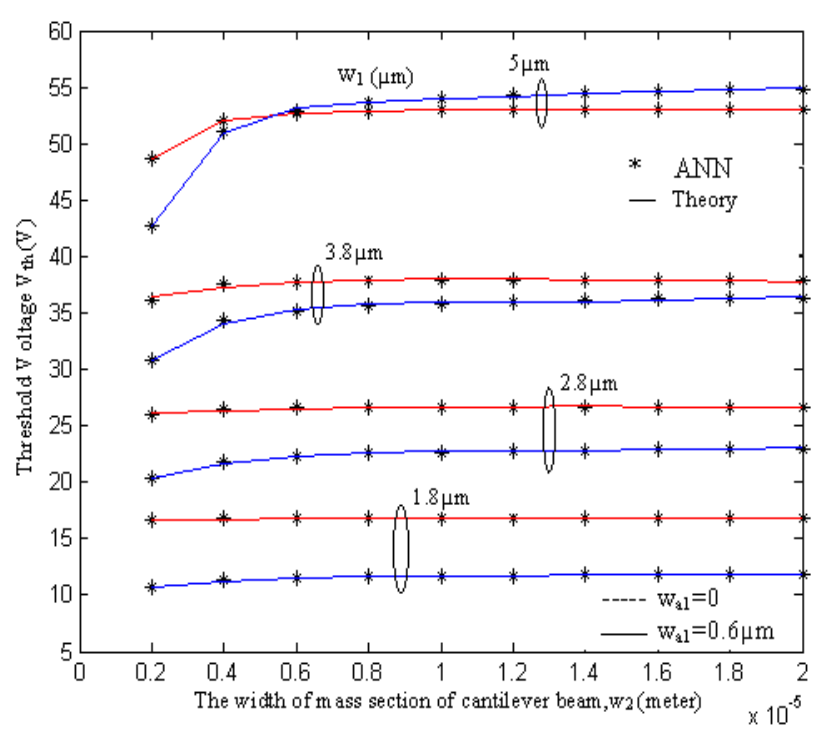

(b)

Fig.6. Comparison of ANN and simulated results of threshold voltage $V_{\text {th }}$ with (a) Various length ratio of $l_{1} /\left(l_{1}+l_{2}\right)$ and initial gap distance $\left(g_{0}\right)$ and (b) Various beam widths $\left(w_{1}, w_{2}\right)$ and Al thickness at sidewalls $\left(w_{A l}\right)$.

Two parameters namely correlation coefficient and Mean Square Error (MSE) values were used for the performance evaluation of the models and comparison of the results for prediction of Sparameters. The higher value of correlation coefficient and a smaller value MSE means, better the performance of the model. The results of the neural network based modeling of S-parameter calculation of different combination of input parameters with the used data set are provided in tables 1 in terms of the correlation coefficient and mean square errors. From fig. $5 \& 6$ and the table 1 , it is observed that there is an excellent agreement between the ANN \& theory result.

\begin{tabular}{|c|c|c|c|c|}
\hline $\begin{array}{c}\left(\mathrm{g}_{0}\right) \\
(\mu \mathrm{m})\end{array}$ & \multicolumn{2}{|c|}{$\begin{array}{l}l_{1} /\left(l_{1}+l_{2}\right)= \\
440 \mu \mathrm{m}\end{array}$} & \multicolumn{2}{l|}{$\begin{array}{l}l_{1} /\left(l_{1}+l_{2}\right)= \\
500 \mu \mathrm{m}\end{array}$} \\
\cline { 2 - 5 } & MSE & $\mathrm{R}^{2}$ & $\mathrm{MSE}$ & $\mathrm{R}^{2}$ \\
\hline 4 & 0.0137 & 0.8099 & 0.0228 & 0.7889 \\
\hline 6 & 0.0013 & 0.9999 & 0.0034 & 0.9987 \\
\hline 8 & 0.0159 & 0.9569 & 0.0114 & 0.9231 \\
\hline 10 & 0.0477 & 0.8899 & 0.0870 & 0.6879 \\
\hline
\end{tabular}

\begin{tabular}{|c|c|c|c|c|}
\hline \multirow{2}{*}{$\begin{array}{c}w_{1} \\
(\mu \mathrm{m})\end{array}$} & \multicolumn{2}{|c|}{$w_{A l}=0 \mu \mathrm{m}$} & \multicolumn{2}{c|}{$w_{A l}=0.6 \mu \mathrm{m}$} \\
\cline { 2 - 5 } & $\mathrm{MSE}$ & $\mathrm{R}^{2}$ & $\mathrm{MSE}$ & $\mathrm{R}^{2}$ \\
\hline 1.8 & 0.0224 & 0.7884 & 0.0169 & 0.8777 \\
\hline 2.8 & 0.0202 & 0.7381 & 0.0895 & 0.6996 \\
\hline 3.8 & 0.1301 & 0.4365 & 0.5012 & 0.8759 \\
\hline 5 & 0.0027 & 0.9998 & 0.0147 & 0.9411 \\
\hline
\end{tabular}

(a)

Table.1. Calculated Mean Square Error (MSE) and Correlation Coefficient $\left(R^{2}\right)$ for threshold voltage $V_{\text {th }}$ (a) MSE and $\left(R^{2}\right)$ with various length ratios (b) MSE and $\mathrm{R}^{2}$ with various beam widths the network trained for mechanical modeling.

The neural network provides better results in terms of the higher correlation coefficient, which measures the strength and direction of the linear relation between two variables (actual and predicted values). The correlation coefficient of $\mathrm{R}=0.9999$ (0.9998) with minimum mean square error (MSE) of the model of 0.0013 ( 0.0027$)$ has been observed for threshold voltage calculation in terms of various length ratios and beam widths. 


\section{CONCLUSION}

We have made a comprehensive analytical S-parameter model based on MLP neural network for RF and mechanical modeling of the series lateral RF MEMS switch which has broad application in high frequency transmitting/receiving circuit, true time delay phase shifter and switching matrix. The double beam lateral switch for up to $25 \mathrm{GHz}$ frequency application has been simulated using neural network. The developed neural model matches quite closely with the literature results. The simulation results shows that the switch have low insertion $(<1 \mathrm{~dB})$ and high isolation $(>10 \mathrm{~dB})$ loss. The threshold voltage can be reduced by reducing the initial gap distance between the electrodes and by providing Aluminum sidewall which increase the electrostatic force which is inverse proposal to threshold voltage. Since the neural models presented in this work have good accuracy, require no tremendous computational efforts and less background information about bridges, they can be very useful for the development of fast CAD algorithms. A distinct advantage of neural computation is that, after proper training, a neural network completely bypasses the repeated iterative processes when new cases are presented to it. Since the training and testing time is less than few microseconds, the proposed neural model is quite fast in the design and loss analysis of lateral MEMS series switch.

\section{REFERENCES}

[1] Muldavin J B and Rebeiz G M, "High isolation MEMS shunt switches part 1: Modeling" IEEE Trans Microwave Theory Tech., 48, pp.1045-1052.

[2] K.E.Peterson, "Micromechanical membrane switches on silicon, "IBM J.Res.Develop.,Vol.23,no.4,pp.376-385,July 1979.

[3] M. Tang, A.Q. Liu, A. Agarwal, Z.-S. Liu, C. Lu, "A single-pole double-throw (SPDT) circuit using lateral metalcontact micro machined switches", IEEE Trans Sensors and Actuators 121, pp.187-196, 2005.

[4] Mohammad N. Mollah and Nema1 C. Karmakar, "RF- MEMS Switches: Paradigms of Microwave Switching", Proceedings of APMC200 1, Taipei, Taiwan, R.O.C.pp.1024-1027, 2001.

[5] Gabriel M. Rebeiz,"RF MEMS Switches: Status of the Technology", Proceedings of the 12th International Conference on Solid State Sensors, Actuators and Microsystems, Boston, June 8-12, pp.1726-1729, 2003.

[6] A Q Liu1, M Tang, A Agarwal and A Alphones, "Low-loss lateral micro machined switches for high frequency applications", J. Micromech. Microeng. 15, pp.157-167, 2005.

[7] Hah D, Yoon E and Hong S, "A low-voltage actuated micro machined microwave switch using torsion springs and leverage",IEEE Trans. Microw. Theory Tech. 48 2540-6, 2000.

[8] G. M. Rebeiz, "RF MEMS, Theory, Design and Technology", John Wiley and Sons, Hoboken, 2003.

[9] Elliot R. Brown, "RF-MEMS switches for reconfigurable integrated circuits", IEEE Trans. Microwave Theory Tech., pp.1868-1880, Nov. 1998.

[10] B.Lakshanarayana, T.Weller, "Distributed MEMS phase shifters on silicon using Tapered Impedance unit cells", in IEEE MTT-S Digest pp.1237-1240, 2002.

[11] T.Shigemastsu,N.Suematsu,N.takeuchi,Y.Iyama,A.Mizobuch, "A 6-18GHz 20W SPDT switch using shunt discrete Pin diodes”, Proceedings of the IEEE MTT-S Dig, pp. 527-530, 1997.

[12] Winchester, K.J.Dell, J.M, "Finite element analysis of tunable fabry perot MEMS structures",Proc. of Optoelectronics and micro electronics materials and devices, pp:324-327,2000.

[13] S.Suganthi, K.Murugesan and S.Raghavan, "Neural Network based Realization and Circuit Analysis of Lateral RF MEMS Series Switch," Proceedings of the IEEE International Conference on Computer,Communication and Electrical Technology, March 18-19, pp.260-265, 2011.

[14] Ai Qun Liu, Aibin YuMing Tang, Muhammad Faeyz Karim and Selin Teo Hwee Gee, "RF MEMS Switches and Integrated Switching Circuits-Design, Fabrication, and Test”, Springer MEMS Reference Shelf, 2010.

[15] Gere J M and Timoshenko S P, "Mechanics of Materia",l 4th edn (Boston, MA: PWS), 1997.

[16] Gregory L. Creech, Bradley J. Paul, Christopher D. Lesniak, Thomas J. Jenkins, Mark C. Calcatera, "Artificial Neural Networks for Fast and Accurate EM-CAD of Microwave Circuits", IEEE Trans. Microwave theory Tech., vol. 45 no.5, pp. $1794-802,1997$.

[17] Younjae Lee and dejan S Filipovic, "ANN based electromagnetic models for the design of RF MEMS switches", IEEE Microwave and Wireless components Letters, Vol.15, No.11, pp.823-825, 2005. 
[18] Zaabab, Q. Zhang, and M. Nakhla, "Analysis and optimizations of microwave circuits and devices using neural networks models", IEEE MTT-S Dig., San Diego., CA, pp. 393 -396, 1994.

[19] S. Haykin 1994, "Neural Networks: A Comprehensive Foundation", Macmillan College Publishing Comp.

[20] K. Levenberg,"A Method for the solution of Certain Nonlinear Problems in Least Squares”, Quart. Appl.Math.Vol. 2, pp.164-168, 1944.

[21] D.J.C. MacKay, 1992, "Bayesian interpolation”, Neural Computation, Vol. 4, pp. 415-447.

[22] C. Yildiz and M. Turkmen, "A CAD Approach Based on Artificial Neural Networks for Shielded Multilayered Coplanar Waveguides”, AEU International Journal of Elect. And Com. Vol. 58, pp.284-292, 2004. 